\title{
OCULAR POINT OF CARE ULTRASOUND (POCUS) DETECTION OF RETINOBLASTOMA IN THE PEDIATRIC EMERGENCY DEPARTMENT
}

Shirley Friedman MD, Miguel M Glatstein MD, Oren Tavor MD, Tali Capua MD, Neta Cohen MD, Ayelet Rimon MD

\section{Background}

Pediatric emergency department (PED) physicians frequently encounter children with symptoms that warrant ocular fundoscopic evaluation. Lack of cooperation, ocular or periorbital trauma, contraindications to mydriasis and photophobia may hinder direct fundoscopic evaluation. Bedside PED ocular point of care ultrasound is a useful tool in the evaluation of the eye and enables the visualization of the ocular anatomy. It is radiation free, rapid, low cost, does not require mydriasis or sedation and is performed on closed eyelids with minimal or no cooperation from the patient. Several publications have described the application of ocular POCUS to diagnose ocular and orbital anomalies such as trauma and orbital masses.

\section{Aim}

We aim to demonstrate the feasibility and efficacy of POCUS in assessing ocular complaints by PED physicians.

\section{Method}

Clinical case presentation.

\section{Results}

A 2 months old baby boy presented to the PED after his parents noticed right leucocoria in a cellular phone photograph. The baby was irritable when fundoscopy was attempted hence ocular POCUS was performed using a linear transducer (Phillips CX50, L8-12 MHz) and demonstrated a right eye retinal mass nasal to the optic disc (Fig 1). The patient was referred to an ophthalmologist who confirmed the diagnosis of Retinoblastoma. The patient underwent MRI yielding the same diagnosis (Fig 2) followed by surgery within 2 weeks of the PED visit.

Figure 1:

POCUS demonstrating right eye retinal mass nasal to the optic disc.

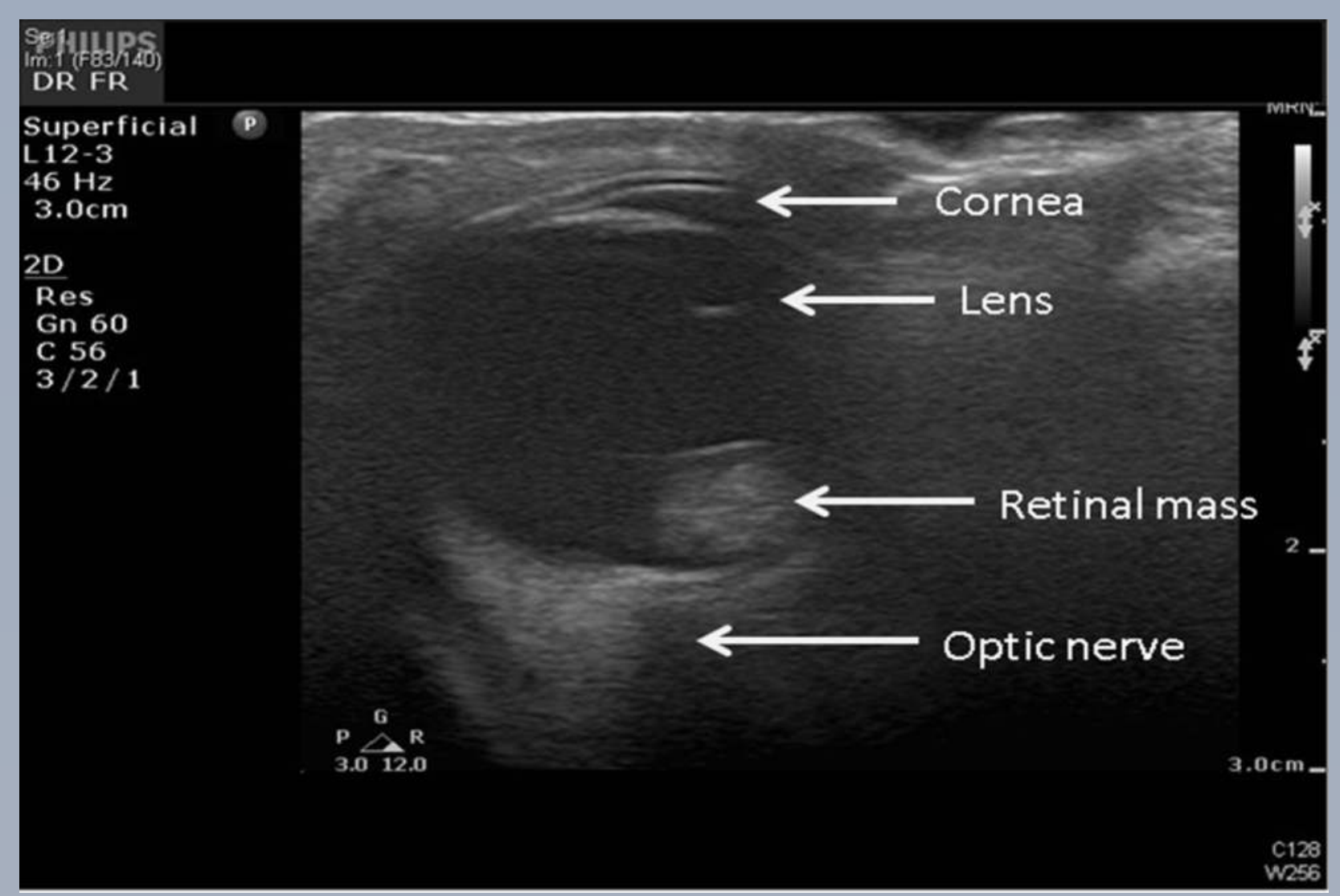

Figure 2:

The retinal mass on MRI.

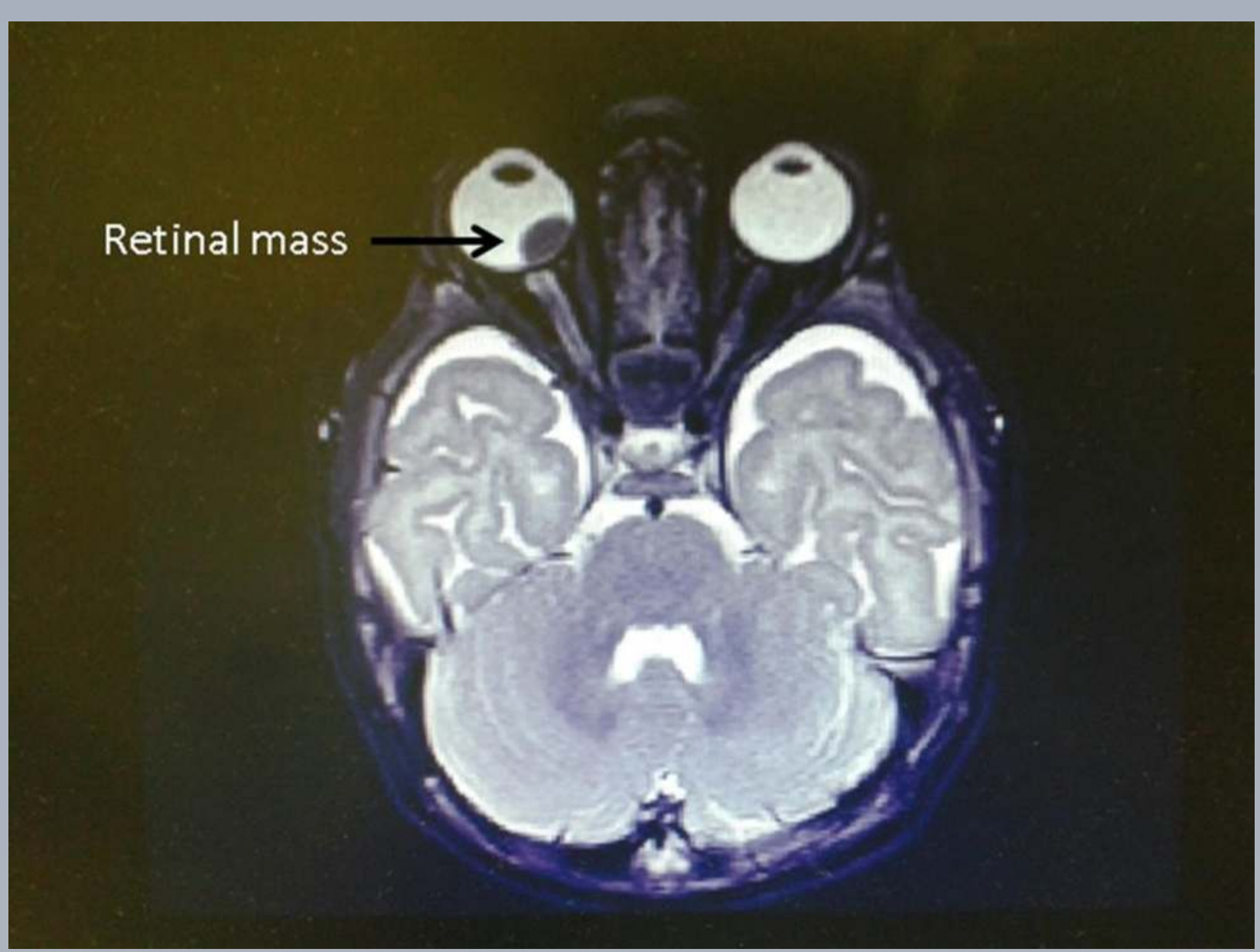

\section{Conclusion}

We suggest that ocular POCUS is safe, fast and easy to perform in the PED setting in uncooperative patients and facilitates rapid, accurate diagnosis and treatment. 